5 Byington CL, Samore MH, Stoddard GJ, et al. Temporal trends of invasive disease due to Streptococcus pneumoniae among children in the intermountain west: emergence of nonvaccine serogroups. Clin Infect Dis 2005;41:21-9.

\section{Repeated tuberculin testing does not induce false positive ELISPOT results}

The Enzyme Linked ImmunoSpot (ELISPOT) is a new rapid $\mathrm{T}$ cell based blood test (otherwise known as an interferon- $\gamma$ assay) for the diagnosis of latent tuberculosis infection. ${ }^{1-3}$ The commercially available form of the assay, T-SPOT ${ }^{\circledR} \mathrm{TB}$ (Oxford Immunotec Abingdon, UK) has European regulatory approval as an in vitro diagnostic test and is increasingly being used in clinical practice. The test is based on the enumeration of interferon- $\gamma$ producing $\mathrm{T}$ cells which are specific for two highly antigenic proteins, early secretory antigenic target-6 (ESAT-6) and culture filtrate protein 10 (CFP-10). These proteins are expressed by Mycobacterium tuberculosis but are absent from $M$ bovis BCG vaccine. Hence, the test does not give false positive results in BCG vaccinated individuals. ${ }^{1-3}$

ESAT- 6 and CFP-10 are, however, contained within tuberculin purified protein derivative (PPD). Since ELISPOT is a highly sensitive method for measuring even low numbers of antigen specific T cells, ${ }^{4}$ concerns have been raised as to whether repeated tuberculin skin tests might induce $\mathrm{T}$ cell responses to these specific antigens, resulting in false positive ELISPOT results.

As T-SPOT ${ }^{\circledR}$ TB enters clinical practice, it may initially be used by some people in conjunction with the tuberculin skin test. It is therefore important to know whether false positive ELISPOT results are induced by tuberculin testing. The following result strongly suggest that this is not the case.

The results reported here are from a 2 year follow up of a group of people with potential point source exposure to multidrug resistan tuberculosis on a maternity unit in Modena University Hospital, Italy. ${ }^{5}$ Forty four BCG unvaccinated subjects were negative at initial screening by tuberculin skin test and ELISPOT, 3 months after the point source exposure ceased. All participants had negative results on serological testing for HIV infection. Tuberculin skin tests were administered and read by two experienced chest physicians using 5 units of PPD-S injected intradermally about 2 hours after blood was drawn for ELISPOT assays. The ELISPOT assays were performed and scored, as previously described, ${ }^{5}$ by two technicians without knowledge of personal identifiers. All these individuals underwent repeated testing by skin test and ELISPOT at 9, 15 and 24 months after the point exposure. At 24 months all 44 individuals remained ELISPOT negative, although three had become positive with the tuberculin skin test (fig 1). Thus, inoculation of three PPD skin tests over a 21 month period in 44 initially ELISPOT negative individuals did not induce any false positive ELISPOT results.

These results show that repeated tuberculin skin testing over time does not induce a $\mathrm{T}$ cell response to ESAT-6 or CFP-10 resulting in false positive ELISPOT results. Our findings suggest that this new interferon- $\gamma$ blood assay could be used in association with the standard PPD skin test without any reduction in its high diagnostic specificity. Given the

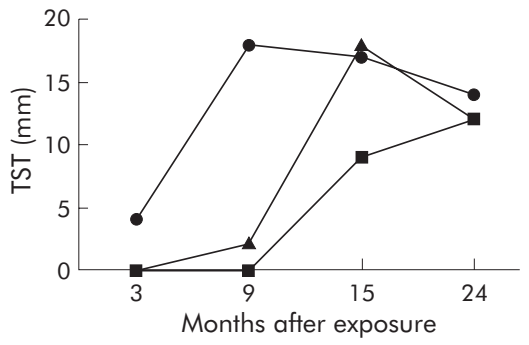

Figure 1 Time course of development of positive Mantoux results in the three participants who became tuberculin skin test (TST) positive as a result of repeated skin testing.

high sensitivity of the ELISPOT assay for detecting even low numbers of antigen specific $\mathrm{T}$ cells, the absence of a detectable response to ESAT- 6 and CFP-10 suggests that $\mathrm{T}$ cells specific for these antigens were not induced by repeated inoculation of PPD. This is consistent with the observation that ESAT6 has very poor immunogenicity when administered as a candidate vaccine, unless inoculated with powerful adjuvants. ${ }^{6}$ This is in stark contrast to its potent immunogenicity when presented to the immune system during natural $M$ tuberculosis infection; indeed, ESAT- 6 is the strongest known target of $\mathrm{T}$ cell responses during tuberculosis infection.

Our results also suggest that T-SPOT ${ }^{\circledR} \mathrm{TB}$ could be especially useful in distinguishing true latent tuberculosis infection from false positive tuberculin skin test results that have arisen through "boosting". Boosting occurs in people who undergo repeated tuberculin skin tests (such as healthcare workers) and causes false positive skin test results in uninfected people. This phenomenon is a major problem in tuberculosis screening programmes for healthcare workers, prisoners, and other groups at persistent risk of tuberculosis exposure, and was almost certainly the reason why three individuals in our study developed positive skin test results after repeated testing. Our findings suggest that $\mathrm{T}$-SPOT ${ }^{\circledR} \mathrm{TB}$ will maintain its high specificity even in individuals with false positive skin test results due to boosting from repeated tuberculin testing. Thus, use of $\mathrm{T}$ SPOT ${ }^{\circledR} \mathrm{TB}$ could enhance our ability to screen for latent tuberculosis infection even in populations who have already been repeatedly screened by the skin test.

L Richeldi

Respiratory Disease Clinic, University of Modena and Reggio Emilia, and Azienda Ospedaliera Policlinico di Modena, Modena, Italy

K Ewer

Nuffield Department of Clinical Medicine, University of Oxford, John Radcliffe Hospital, Oxford, UK

M Losi, P Roversi, L M Fabbri

Respiratory Disease Clinic, University of Modena and Reggio Emilia, and Azienda Ospedaliera Policlinico di Modena, Modena, Italy

A Lalvani

Nuffield Department of Clinical Medicine, University of Oxford, John Radcliffe Hospital, Oxford, UK

Correspondence to: Dr A Lalvani, Nuffield Department of Clinical Medicine, University of Oxford, John Radcliffe Hospital, Oxford OX3 9DU, UK ajit.lalvani@ndm.ox.ac.uk
The study was approved by the Modena research ethics committee and each study participant provided written informed consent.

doi: 10.1136/thx.2005.049759

This work was supported by the Wellcome Trust and Azienda Ospedaliera Policlinico di Modena.

Competing interests: $\mathrm{AL}$ is a named inventor on patents relating to $T$ cell based diagnosis filed by the University of Oxford. Regulatory approval and commercialisation of ELISPOT (T-SPOT TB) has been undertaken by a spin out company of the University of Oxford (Oxford Immunotec Ltd), in which AL has a share of equity and to which he acts as scientific advisor in a non-executive capacity. KE is a named inventor on a patent application relating to the application of ELISPOT filed by the University of Oxford. The University of Oxford has a share of equity in Oxford Immunotec Ltd.

\section{References}

1 Lalvani A, Pathan AA, McShane $\mathrm{H}$, et al. Rapid detection of Mycobacterium tuberculosis infection by enumeration of antigen-specific T cells. Am J Respir Crit Care Med 2001;163:824-8.

2 Lalvani A, Pathan AA, Durkan H, et al. Enhanced contact tracing and spatial tracking of Mycobacterium tuberculosis infection by enumeration of antigen-specific T cells. Lancet 2001;357:2017-21.

3 Ewer K, Deeks J, Alvarez L, et al. Comparison of T-cell-based assay with tuberculin skin test for diagnosis of Mycobacterium tuberculosis infection in a school tuberculosis outbreak. Lancet 2003;361: 1168-73.

4 Lalvani A, Brookes R, Hambleton S, et al. Rapid effector function in CD8+ memory T cells. J Exp Med 1997; 186:859-65.

5 Richeldi L, Ewer K, Losi M, et al. T cell-based tracking of multidrug resistant tuberculosis infection after brief exposure. Am J Respir Crit Care Med 2004:170:288-95.

6 Brandt L, Elhay M, Rosenkrands I, et al. ESAT-6 subunit vaccination against Mycobacterium tuberculosis. Infect Immun 2000;68:791-5.

\section{Clinical importance of the Step 3 choice in asthma}

We read with interest the meta-analysis by Masoli et al ${ }^{1}$ which aimed to further guide clinicians in their choice between addition of long acting $\beta_{2}$ agonists (LABA) or use of higher doses of inhaled corticosteroids (ICS) in patients with symptomatic asthma. The pooled odds of at least one moderate or severe exacerbation was 1.35 times higher in those receiving a higher dose of ICS than in those treated with LABA.

Unfortunately, it is difficult to draw any meaningful conclusion as to the clinical relevance of these findings or to compare at a glance the results with those of the previous MIASMA study ${ }^{2}$ because of differences in the summary statistics presented. For clinicians to understand the clinical context of these two studies, it is helpful to calculate the number needed to treat (NNT), as was done in the original MIASMA study.

Of the 2312 patients randomised to LABA treatment included in the newer study, 184 experienced one or more moderate or severe exacerbations (an incidence of 79.6 per 1000 patients) compared with 243 of the 2264 patients randomised to high dose ICS treatment (an incidence of 107.3 per 1000 patients). These incidences give an attributable risk reduction of 27.7 per 1000 patients which represents an NNT of 37, meaning that for every 37 patients receiving LABA in preference to high dose ICS, one less will experience an exacerbation. The corresponding 